\title{
InSAR data for monitoring land subsidence: time to think big
}

\author{
A. Ferretti, D. Colombo, A. Fumagalli, F. Novali, and A. Rucci \\ TRE - Tele-Rilevamento Europa, Milano, Italy \\ Correspondence to: A. Ferretti (alessandro.ferretti@treuropa.com)
}

Published: 12 November 2015

\begin{abstract}
Satellite interferometric synthetic aperture radar (InSAR) data have proven effective and valuable in the analysis of urban subsidence phenomena based on multi-temporal radar images. Results obtained by processing data acquired by different radar sensors, have shown the potential of InSAR and highlighted the key points for an operational use of this technology, namely: (1) regular acquisition over large areas of interferometric data stacks; (2) use of advanced processing algorithms, capable of estimating and removing atmospheric disturbances; (3) access to significant processing power for a regular update of the information over large areas. In this paper, we show how the operational potential of InSAR has been realized thanks to the recent advances in InSAR processing algorithms, the advent of cloud computing and the launch of new satellite platforms, specifically designed for InSAR analyses (e.g. Sentinel-1a operated by the ESA and ALOS2 operated by JAXA). The processing of thousands of SAR scenes to cover an entire nation has been performed successfully in Italy in a project financed by the Italian Ministry of the Environment. The challenge for the future is to pass from the historical analysis of SAR scenes already acquired in digital archives to a near real-time monitoring program where up to date deformation data are routinely provided to final users and decision makers.
\end{abstract}

\section{Introduction}

Results from satellite InSAR make it a unique instrument for ground displacement analysis, which contributes to the portfolio of technologies of traditional monitoring techniques and can be used in many diverse applications (Ferretti, 2014). InSAR data can be used for fault characterization and calibration of geo-mechanical models in the oil and gas sector, for monitoring landslides, volcanoes and seismic faults, areas prone to sinkholes and subsidence, terrain compaction phenomena induced by tunneling works, and even for monitoring the stability of individual buildings.

Although the first results date back to the 1980's (Gabriel et al., 1989), InSAR is often seen as a "new technology" for surface deformation monitoring and the full potential of InSAR is largely under-recognized, even within the scientific community. The reasons behind this slow uptake are numerous and complex (Ferretti, 2014). In the following sections, it will be shown how most of the challenges related to an operational use of InSAR data (e.g. monitoring urban subsidence) have now been overcome and why this technol- ogy can now become a standard tool for studying subsidence and other geological processes.

\section{The space segment}

Knowledge dissemination of any space technology is strongly related to the availability of a reliable and robust space segment, providing proper and adequate infrastructure and data sources. For InSAR, despite the results from SEASAT (1978), the Sentinel-1A European Space Agency (ESA) SAR satellite that entered its operational phase in late 2014 is the first (civilian) sensor specifically designed for surface deformation monitoring over large areas. So far, all SAR sensors have been multi-purpose, where repeat-pass InSAR was just one of a long list of possible applications, from iceberg monitoring and oil-spill detection, to soil moisture estimation and biomass mapping.

The first InSAR results from satellite sensors were a somewhat unexpected, although extremely welcome, outcome (Gabriel et al., 1989). However, after obtaining proof of con- 
cept with SEASAT and the success of the ERS missions operated by ESA, InSAR technology still did not take-off as fast as expected. The space segment aspects of the satellite missions did not aid its evolution either. The specifications of satellites being developed in the new millennium were not in the direction required for systematic and repeatable InSAR observations. Radar interferometry needs a simple acquisition scheme, with radar sensors acquiring data regularly over a particular area using the very same acquisition mode and geometry, to allow for comparison of range values over time. SAR technicians and aerospace industries, on the contrary, were focused on a different goal: on offering many sophisticated imaging options, in terms of polarization of the electromagnetic signal wave, image resolution, incidence angle of the radar beam, etc., and not on simple, single-mode radar sensors. Even considering the more recent satellites, the requirement to task the satellite to acquire an image, while selecting the correct acquisition mode (from a long list of options), to create a homogeneous multi-temporal data-set over an area does not facilitate the diffusion of InSAR as a standard geodetic tool (Ferretti, 2014).

Despite the difficulties faced in the last two decades, the launch of the satellite platform Sentinel-1A can be considered a milestone on the path towards an operational use of InSAR. The Sentinel-1 mission, operated by the ESA, has been specifically designed for large-scale InSAR analyses (Torres et al., 2012). Sentinel-1A and the twin sensor Sentinel 1-B (to be launched in 2016) are two C-band, polar-orbiting, satellite systems for the continuation of SAR operational applications, but with significant enhancements, compared to previous ESA missions, in terms of revisitation, coverage, timeliness and reliability of service. The Interferometric Wide Swath acquisition mode can image a $250 \mathrm{~km}$ swath, more than twice that of the ESA ERS mission, with a repeat cycle of 12 days, compared to the 35 days of ERS. Once both sensors will be operational the effective repeat cycle for InSAR analyses will be 6 days (Salvi et al., 2012). Moreover, radar data will be delivered within an hour of acquisition, a big improvement over existing SAR systems.

Apart from the Sentinel-1 mission, it is important to point out that the number of satellite data sources is increasing steadily. Today, SAR data for operational InSAR projects can be provided by RADARSAT-2, Sentinel-1A, RISAT1, ALOS-2, TerraSAR-X, KOMPSAT-5, Tandem-X and the COSMO-SkyMed constellation (4 SAR satellites). Although not operating with the same central frequency and the same acquisition modes, all these sensors can be used successfully for monitoring surface deformation phenomena. This scenario allows users to design redundant acquisition schemes, where the possible failure of a satellite sensor may not critically compromise the monitoring project. A more "InSARfriendly" and robust space segment will be a key factor for a large scale application of InSAR in the near future.

\section{New algorithms and a case history}

Even within the geodetic community, the slow uptake of SAR interferometry has been interpreted as evidence of the fact that InSAR is still not a mature technology. InSAR analyses are thought to provide interesting but qualitative data, great subjects to write scientific papers, but with a limited impact on real-life applications.

In our opinion, this interpretation is incorrect and overlooks an important point: compared to what was possible in the 1990's, InSAR algorithms have significantly improved, allowing a much better estimation of the signal of interest, constantly reducing error and computational times. The development of Persistent Scatterer Interferometry (Ferretti et al., 2001, 2011; Kampes, 2006) has made it possible to extract millimeter-accuracy displacement data over millions of measurement points automatically identified by a statistical analysis operated over long temporal series of radar images acquired over the same area of interest. The impact of atmospheric disturbances on InSAR data can be strongly reduced. Validation of Persistent Scatterer (PS) data have been reported in dozens of scientific papers (see Ferretti, 2014 and references therein) and large-scale applications have been proven feasible and effective (Costantini et al., 2015).

A good example of the potential related to satellite radar data was a large-scale InSAR project financed by the Italian Ministry of the Environment. In 2007, the Italian Government began supporting the largest ever InSAR analysis to be funded by a national government. The project was designed to map unstable areas over the entire Italian territory, using PS data. In January 2008, the Italian Ministry of the Environment awarded a contract to the industrial consortium, composed of TRE, e-geos and Compulab (Costantini et al., 2015). The main project objective was to expand and update the National Cartographic Portal (PCN) with displacement data for 1992-2010. The project involved processing the entire ESA ERS and Envisat SAR image archives over Italy ( $>15000$ SAR images covering $>330000 \mathrm{~km}^{2}$ ), which called for a dedicated geospatial database to manage millions of PS measurements, thousands of SAR images and hundreds of interferometric data-stacks (Fig. 1). All radar acquisitions have been processed successfully and geologists and geophysicists in central and local administrations can now use PS data to get qualitative and quantitative information about a range of phenomena, affecting known and new at-risk areas, including:

- slow-moving landslides that could trigger fast-moving slides,

- volcanism and related dynamics,

- subsidence and compaction,

- seismic and aseismic fault movement,

- other known and previously unidentified phenomena. 


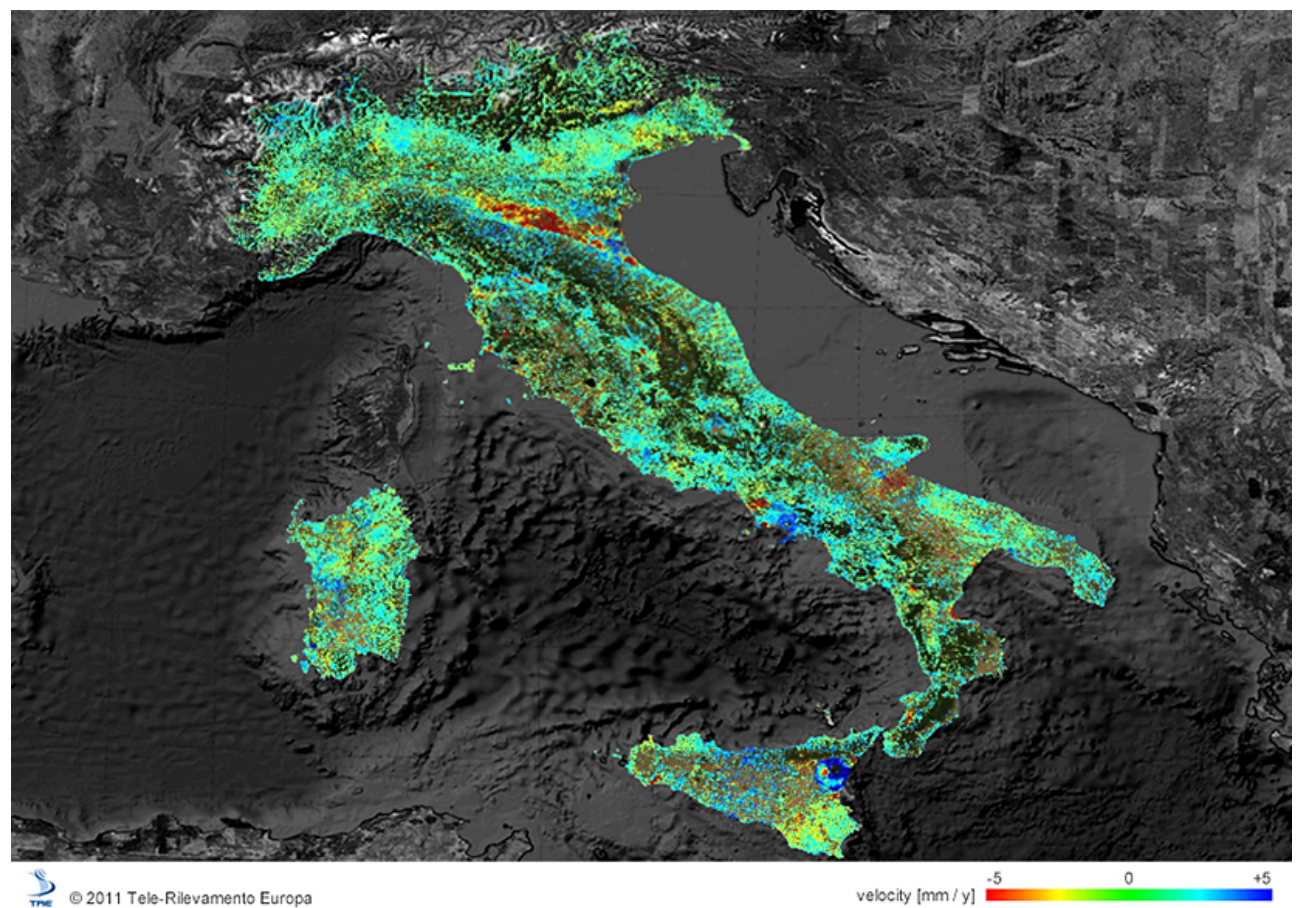

Figure 1. Large-scale mapping of unstable areas over the entire Italian territory. More than 8000 ESA ERS satellite images were processed. Colored dots correspond to measurement points. Red areas are affected by subsidence. The total area covered is $330000 \mathrm{~km}^{2}$. (Radar data: ERS, June 1992-June 2000. Background image: Google Earth ${ }^{\mathrm{TM}}$.)

Moreover, displacement data from medium-resolution ESA SAR data are now being updated using high-resolution COSMO-SkyMed imagery, allowing the identification of a much higher density of measurement points and a larger temporal frequency of acquisitions.

This case history could serve as an example for other nations to exploit the Sentinel radar images (or any other data sources) to create a national database of terrain movements. In general, there is growing interest in Europe across a wide range of topics in which satellite InSAR data could play a role, including: subsidence and its role in relative sea-level rise along coastal areas, induced seismicity (due to oil and gas extraction and fluid injection), shale gas exploitation, sinkhole detection and pipeline monitoring. Assuredly, the regular, long-term acquisition of InSAR data will prompt new areas of scientific exploration and stimulate new commercial applications and market sectors.

The experience related to this successful project has shown that InSAR is a mature technology and how the new processing algorithms can pave the way to new applications. Depending on the processing technique and the resolution of the SAR sensor, InSAR can be used for monitoring displacement of individual structures, but also to provide information at regional and at larger scales. The ability to detect ground displacements that have occurred over hundreds or thousands of square kilometres and even at a national scale can be extremely useful in characterizing and addressing areas prone to risk. InSAR data can allow Civil Protection authorities to analyze and evaluate different scenarios and to plan specific actions based on homogeneous and reliable measurements. Last, but not least, InSAR data can be updated regularly, as more SAR images are acquired by satellite sensors, and can provide new ways to design early warning systems covering entire nations. Also InSAR can identify specific areas where in situ sensors and continuous monitoring tools should be installed. In our opinion, rather than replacing conventional technologies, in the future satellite data will work more in synergy with airborne sensors and ground-based instruments.

\section{Processing capabilities and future trends}

Data processing and storage capabilities are keeping pace with the growing number of information sources. The power of cloud computing is already impacting on remote sensing projects. Customers are becoming impatient with the delayed availability of processed Earth observation data and are increasingly demanding near real-time results from monitoring systems. This will definitely become possible thanks to distributed computing. Surely, displacement data will become available soon after any new satellite acquisition.

Another recent trend in InSAR monitoring projects is the use of different SAR data sources (e.g. Sentinel-1 and COSMO-SkyMed) to increase the number of acquisitions per year over an area of interest. Although no useful interfero- 
gram can be generated using two radar sensors having different frequencies and orbits, nevertheless, their displacement measurements can be combined, whenever the incidence angle of their radar beams do not differ significantly from one another (a tolerance of a few degrees is usually acceptable). It is then possible to sample the signal of interest (i.e. the displacement field) with a temporal frequency much higher than that of the repeat orbit for individual sensors: the concept of a virtual constellation, where different satellite data sources are used in synergy to create a more effective and robust Earth observation system. For instance, one could envision a monitoring scheme with two radar sensors: one operating at L-band (e.g. ALOS-2; $23 \mathrm{~cm}$ wavelength) and one at C-band (e.g. Sentinel-1; $6 \mathrm{~cm}$ wavelength) where InSAR results of the longer wavelength sensor are used to constrain phase unwrapping procedures on interferograms generated using the smaller wavelength sensor (more sensitive to any target displacement and therefore generating interferometric fringes having a higher spatial frequency).

In general, the use of real and virtual satellite constellations will significantly increase the frequency of observation of an area of interest and will make any monitoring project more robust by mitigating consequences of satellite failure. In fact, a very unwelcome event during an InSAR monitoring project is the lack of satellite acquisitions due to satellite maneuvers (orbit correction), conflicts with higher priority requests (for dual-use systems), and temporary (or permanent) failure of the radar sensor or the downlink system. The larger the number of data sources, the more robust the acquisition scheme and the higher likelihood of good data coverage for the entire project duration.

\section{Conclusions}

InSAR is a multi-scale tool: depending on the processing technique and the resolution of the SAR sensor, it can be used for monitoring individual structures, but also to provide information at regional and at larger scales. Having the ability to detect ground displacements that have occurred over hundreds or thousands of square kilometres and even at a national scale can be extremely useful in characterising and addressing areas prone to risk, such as highlighting areas affected by subsidence phenomena. InSAR data can allow decision makers to analyse and evaluate different scenarios and to plan specific actions based on homogeneous and reliable measurements. InSAR data can be updated regularly, as more SAR images are acquired by satellite sensors, and can provide new ways to design early warning systems covering entire nations, where satellite information can highlight areas where in situ sensors and continuous monitoring tools should be installed. Rather than replacing conventional technologies, InSAR data will work more in synergy with airborne sensors and ground-based instruments.
Acknowledgements. The authors thank all TRE colleagues for continuing support, Fabio Rocca and Claudio Prati of Politecnico di Milano for many stimulating discussions on the applications of InSAR data, as well as the two reviewers who improved significantly the clarity of this paper.

\section{References}

Costantini, M., Minati, F., Ciminelli, M. G., Ferretti, A., and Costabile, S.: Nationwide Ground Deformation Monitoring by Persistent Scatterer Interferometry, IEEE Geoscience and Remote Sensing Society, the International Geoscience and Remote Sensing Symposium 2015 (IGARSS 2015), 25-31 July 2015, Milano, Italy, 2015.

Ferretti, A.: Satellite InSAR Data: Reservoir Monitoring from Space, EAGE Publications bv, 2014.

Ferretti, A., Prati, C., and Rocca, F.: Permanent Scatterers in SAR Interferometry, IEEE T. Geosci. Remote, 39, 8-20, 2001.

Ferretti, A., Fumagalli, A., Novali, F., Prati, C., Rocca, F., and Rucci, A.: A new algorithm for processing interferometric datastacks: SqueeSAR, IEEE T. Geosci. Remote, 49, 3460-3470, 2011.

Gabriel, A. K., Goldstein, R. M., and Zebker, H. A.: Mapping small elevation changes over large areas: differential radar interferometry, J. Geophys. Res., 94, 9183-9191, 1989.

Kampes, B. M.: Radar Interferometry - Persistent Scatterer Technique, Springer, 43-69, 2006.

Salvi, S., Stramondo, S., Funning, G. J., Ferretti, A., Sarti, F., and Mouratidis, A.: The Sentinel-1 mission for the improvement of the scientific understanding and the operational monitoring of the seismic cycle, Remote Sens. Environ., 120, 164-174, 2012.

Torres, R., Snoeij, P., Geudtner, D., Bibby, D., Davidson, M., Attema, E., Potin, P., Rommen, B., Floury, N., Brown, M., Navas Traver, I., Deghaye, P., Duesmann, B., Rosich, B., Miranda, N., Bruno, C., L'Abbate, M., Croci, R., Pietropaolo, A., Huchler, M., and Rostan, F.: GMES Sentinel-1 mission, Remote Sens. Environ., 120, 9-24, 2012. 